\title{
Stand Establishment and Persistence of Perennial Cool-Season Grasses in the Intermountain West and the Central and Northern Great Plains
}

\author{
Joseph G. Robins, ${ }^{1}$ Kevin B. Jensen, ${ }^{1}$ Thomas A. Jones, ${ }^{1}$ Blair L. Waldron, ${ }^{1}$ Michael D. Peel, ${ }^{1}$ Craig \\ W. Rigby, ${ }^{2}$ Kenneth P. Vogel, ${ }^{3}$ Robert B. Mitchell, ${ }^{4}$ Antonio J. Palazzo, ${ }^{5}$ and Timothy J. Cary ${ }^{6}$ \\ Authors are ${ }^{1}$ Research Geneticists and ${ }^{2}$ Agronomist, USDA-ARS Forage and Range Research Laboratory, Logan, UT 84322, USA; ${ }^{3}$ Supervisory Research \\ Geneticist and ${ }^{4}$ Research Agronomist, USDA-ARS Grain, Forage, and Bioenergy Research Unit, Lincoln, NE 68583, USA; and ${ }^{5}$ Research Agronomist and \\ ${ }^{6}$ Physical Science Technician, USACE Cold Regions Research and Engineering Laboratory, Hanover, NH 03755, USA.
}

\begin{abstract}
The choice of plant materials is an important component of revegetation following disturbance. To determine the utility and effectiveness of various perennial grass species for revegetation on varied landscapes, a meta analysis was used to evaluate the stand establishment and persistence of 18 perennial cool-season grass species in 34 field studies in the Intermountain and Great Plains regions of the United States under monoculture conditions. Combined across the 34 studies, stand establishment values ranged from $79 \%$ to $43 \%$ and stand persistence values ranged from $70 \%$ to $0 \%$. Intermediate wheatgrass (Thinopyrum intermedium [Host] Barkworth \& D. R. Dewey), tall wheatgrass (Thinopyrum ponticum [Podp.] Z.-W. Liu \& R.-C. Wang), crested wheatgrass (Agropyron spp.), Siberian wheatgrass (Agropyron fragile [Roth] P. Candargy), and meadow brome (Bromus riparius Rehmann) possessed the highest stand establishment $(\geq 69 \%)$. There were no significant differences among the 12 species with the largest stand persistence values. Basin wildrye (Leymus cinereus (Scribn. \& Merr.) Á. Löve), Altai wildrye (Leymus angustus [Trin.] Pilg.), slender wheatgrass (Elymus trachycaulus [Link] Gould ex Shinners), squirreltail (Elymus spp.), and Indian ricegrass (Achnatherum hymenoides [Roem. \& Schult.] Barkworth) possessed lower stand persistence ( $\leq 32 \%)$ than the majority of the other species, and Indian ricegrass $(0 \%)$ possessed the lowest stand persistence of any of the species. Correlations between environmental conditions and stand establishment and persistence showed mean annual study precipitation to have the most consistent, although moderate effect $(r=\sim 0.40)$ for establishment and persistence. This relationship was shown by the relatively poor stand establishment and persistence of most species at sites receiving less than 310 $\mathrm{mm}$ of annual precipitation. These results will be a tool for land managers to make decisions concerning the importance of stand establishment, stand persistence, and annual precipitation for revegetation projects on disturbed sites.
\end{abstract}

Key Words: environmental conditions, grass species, precipitation, revegetation

\section{INTRODUCTION}

Attempts to revegetate degraded lands in western North America began in the early 1900s and resulted in varying levels of success (Pickford 1932). Ecosystem degradation resulted in weakened ecological health and function, including loss of soil resources from erosion and loss of plant species diversity. This fed into the cycle of increasing annual plant invasion and fire disturbance. Invasive annual plants such as cheatgrass (Bromus tectorum L.) create unfavorable conditions for perennial vegetation by changing soil structure and properties (Norton et al. 2004, 2007). Reseeding these disturbed sites with genetically improved cultivars that establish in the presence of undesirable vegetation is often the most effective and economically feasible way to improve such sites (Asay et al. 2003).

Mention of a proprietary product does not constitute a guarantee or warranty of the product by the US Dept of Agriculture (USDA), US Army Corps of Engineers (USACE), or the authors and does not imply its approval to the exclusion of the other products that also might be suitable.

Correspondence: Joseph Robins, USDA-ARS Forage and Range Research Laboratory, 6300 Old Main Hill, Logan, UT 84322, USA. Email: Joseph.Robins@ars.usda.gov

Manuscript received 23 February 2011; manuscript accepted 5 August 2012.

(c) 2013 The Society for Range Management
Revegetation with introduced grasses, such as crested wheatgrass, became common because of better stand establishment, persistence, and weed suppression than perennial native grass species (Aguirre and Johnson 1991; Asay et al. 2001; Thacker et al. 2009). However, due to increasing desire and need for revegetation with perennial native grass species, efforts began to focus on development of improved plant materials of these species (Jones et al. 1991; Asay 1992).

Research entities from various US and state government agencies share a long history of developing and evaluating improved plant materials for rangeland revegetation in the western United States. Among the work carried out over the years, stand establishment and stand persistence data is available from over 30 studies at diverse ecological sites across the Great Plains and Intermountain regions of the United States. Although small components of these studies have been reported previously (Asay et al. 2001; Robins et al. 2007), a comprehensive analysis has not yet been published. Thus, the objectives of this study were to: 1) use a meta analysis to compile and analyze stand establishment and persistence of various plant materials of 18 cool-season, perennial grasses from 34 field studies; 2) determine the effect of climatic and environmental conditions on the stand establishment and persistence of these species; and 3) where possible, evaluate the performance of improved compared to standard plant materials. 


\section{METHODS}

\section{Evaluation Sites and Data Collection}

The dataset evaluated came from 34 field studies located at sites across the Intermountain West and northern and central Great Plains areas of the United States. A few of the sites were used for more than one study (Table 1). Although the repeated use of some sites likely introduced covariance structure into the model, the studies at each site were not identical because studies were separated by years, different varieties were included in the different studies, and the climatic conditions were often very different for the studies at the same site (Table 1). Thus, the covariance created in the model by some repeated use of sites was considered less important than including the additional data from varieties and climatic conditions. The sites were located in seven states in the Intermountain and Great Plains regions of the United States and represented 12 plant adaptation regions (PARs; Vogel et al. 2005). The sites included a wide range of climatic and environmental conditions including precipitation, elevation, latitude, longitude, temperature, and soil type. The data set spans more than $20 \mathrm{yr}$ (19832006).

Typical site preparation consisted of rototilling in the fall for degradation and removal of native plant vegetation, chemical fallow (glyphosate $2.3 \cdot \mathrm{L} \cdot \mathrm{ha}^{-1}$ ) the following summer, and cultivation followed by a roller or harrows prior to seeding in the fall. The majority of sites were seeded using either a John Deere flex planter with depth bands (Deere and Co, Moline, IL) or a Hegge cone seeder with depth control (Wintersteiger, Inc, Salt Lake City, UT). Sites were seeded in fall using a pure live seed seeding rate (based on germination) of one seed linear $\cdot \mathrm{cm}^{-1}$ at a $0.64-\mathrm{cm}$ depth. Using equal numbers of seeds for each species is commonly used in seeding evaluations with multiple species (Asay et al. 2001; Palazzo et al. 2005). Actual seeding rates ranged from $9 \mathrm{~kg} \cdot \mathrm{ha}^{-1}$ (Siberian wheatgrass) to $29 \mathrm{~kg} \cdot \mathrm{ha}^{-1}$ (Altai wildrye) with a mean of $16 \mathrm{~kg} \cdot \mathrm{ha}^{-1}$. The larger-seeded species, such as Altai wildrye, meadow brome, and RS wheatgrass (Elymus hoffmannii K. B. Jensen \& K. H. Asay), had higher seeding rates on a $\mathrm{kg} \cdot \mathrm{ha}^{-1}$ basis. Emergence occurred in fall of the seeding year for the Nebraska sites, or the following spring for all other sites. Plot size varied depending on location, but ranged from approximately $4 \mathrm{~m}^{2}$ to $6 \mathrm{~m}^{2}$. Each trial was comprised of a randomized complete block design with either three or four blocks. Common seed sources could not be used for the same variety in each study due to the span of years involved in the work. However, in all cases seed was tested for germination to ensure proper seed rate on a pure live seed basis.

Stand establishment and stand persistence data were collected from 34 and 22 studies, respectively. Stand establishment was measured the first year after seeding and persistence was measured in the third year after seeding. Values for both measurements pertained just to the seeded species corresponding to each plot and were representative of monocultures. Prior to 1999 , all data were collected using visual estimates of percent stand based on a 1 to 9 rating. Visual estimates were converted to a percentage by increasing the percentage by $12.5 \%$ for each numeric increase $(1=0 \%, 5=50 \%, 9=100 \%)$. Subsequently, the method of Vogel and Masters (2001) was employed. A square frame with interior grids $\left(15 \mathrm{~cm}^{2}\right)$ was used by counting the number of square grids within the frame that contained live, rooted plant material that had been seeded in each plot. This procedure was repeated twice in each plot and the value was then converted to a proportion. Because the visual and grid methods were not used simultaneously at a site, there was no way to estimate the correlation between the two methods.

\section{Plant Materials}

Eighteen cool-season grass species were included at some or all of the studies (Table 2). Not all species were included in each study. The number of species included in a study ranged from 2 to 16 . Species were chosen according to seed availability and their expected adaptation to each site. The species were big squirreltail (Elymus multisetus M. E. Jones), bottlebrush squirreltail (Elymus elymoides [Raf.] Swezey), bluebunch wheatgrass (Pseudoroegneria spicata [Pursh] Á. Löve), basin wildrye, Indian ricegrass, slender wheatgrass, Snake River wheatgrass (Elymus wawawaiensis J. Carlson \& Barkworth), thickspike wheatgrass (Elymus lanceolatus [Scribn. \& J. G. Sm.] Gould), western wheatgrass (Pascopyrum smithii [Rydb.] Á. Löve), Altai wildrye, crested wheatgrass, intermediate wheatgrass, meadow brome, RS wheatgrass, Russian wildrye (Psathyrostachys juncea [Fisch.] Nevski), Siberian wheatgrass, smooth brome (Bromus inermis Leyss.), and tall wheatgrass. Crested wheatgrass and Russian wildrye were the only species included in all studies. Sixty-nine varieties (varieties or germplasms) were included in the analysis (Table 3). With the exception of Kazak Siberian wheatgrass, each of the varieties is officially released from a public or private entity and is (or has been) commercially available for rangeland revegetation.

\section{Data Analysis}

Data were analyzed using mixed model procedures. The main effects of species and variety nested within species (variety) were considered fixed. The main effects of study, blocks nested within study, and all interactions were considered random. The mixed procedure of the SAS Statistical software (Vers. 9.3, SAS, Inc, Cary, NC) was used for analysis. Statistical models for stand establishment and stand persistence were analyzed both within each study and across all studies. Due to nonnormality, data were arcsin-transformed prior to analysis and then reverse-transformed for presentation and discussion.

Least significant differences were estimated for each study individually and for the overall across study analysis. Due to the unbalanced nature of the data, least significant differences were estimated using the standard errors of a mean difference of each species when compared to crested wheatgrass, similar to the proposed methods of Jones (1988) and Dourleijn (1993). Crested wheatgrass was chosen as the check because it was one of only two species included in each study. All discussions of significance are based on a minimum critical value of $\alpha=0.05$ unless otherwise noted.

\section{RESULTS}

\section{Species and Varieties}

Combined across the 34 studies, stand establishment values ranged from $79 \%$ (intermediate wheatgrass) to $43 \%$ (Indian 
Table 1. Environmental and climatic characteristics for the 34 studies from which establishment and persistence data was collected for the species included in the study from 1983-2006.

\begin{tabular}{|c|c|c|c|c|c|c|c|c|c|}
\hline Location & PAR $^{1}$ & $\begin{array}{c}\text { Year } \\
\text { planted }\end{array}$ & Soil type & Latitude & Longitude & $\begin{array}{c}\text { Mean annual } \\
\text { precipitation (mm) }\end{array}$ & $\begin{array}{c}\text { Maximum } \\
\text { temperature }\left({ }^{\circ} \mathrm{C}\right)\end{array}$ & $\begin{array}{c}\text { Minimum } \\
\text { temperature }\left({ }^{\circ} \mathrm{C}\right)\end{array}$ & Elevation $(\mathrm{m})$ \\
\hline \multicolumn{10}{|l|}{ Colorado } \\
\hline \multirow[t]{2}{*}{ Turkey Creek } & GPPDS5 & 1996 & Neville-Rednun complex/ & & & & & & \\
\hline & & & Rizozo-Neville complex & 38.62 & -104.88 & $446(219-593)$ & $16.7(14.8-17.5)$ & $0.9(-0.1$ to 1.4$)$ & 1898 \\
\hline \multicolumn{10}{|l|}{ Idaho } \\
\hline King Hill & IS6 & 2003 & Lankbush-Lanktree complex & 43.07 & -115.14 & $300(292-308)$ & $17.2(16.6-17.8)$ & 3.8 (3.4 to 4.2$)$ & 1131 \\
\hline Malta & IS5 & 1985 & Declo silt loam & 42.30 & -113.20 & $280(210-357)$ & $16.6(14.7-17.4)$ & $0.2(-1.6$ to 0.6$)$ & 1468 \\
\hline Malta & IS5 & 2004 & Declo silt loam & 42.30 & -113.20 & $274(193-376)$ & $16.2(15.7-17.1)$ & $0.4(-0.1$ to 0.9$)$ & 1468 \\
\hline \multirow[t]{2}{*}{ Picabo } & IS5 & 1985 & Gooding-Gooding eroded- & & & & & & \\
\hline & & & Hamrub complex & 43.24 & -114.24 & $330(325-335)$ & $13.4(11.9-14.8)$ & $-3.3(-4.9$ to -1.6$)$ & 1507 \\
\hline Stone & IS5 & 1984 & Mellor-Freedom complex & 42.04 & -112.67 & 337 (295-373) & $15.5(14.2-16.4)$ & $-1.1(-2.5$ to 0.2$)$ & 1408 \\
\hline Stone & IS5 & 1989 & Mellor-Freedom complex & 42.04 & -112.67 & $258(186-339)$ & $16.2(15.4-17.4)$ & $-0.7(-2.1$ to 0.1$)$ & 1408 \\
\hline Stone & IS5 & 2002 & Mellor-Freedom complex & 42.04 & -112.67 & $310(233-482)$ & $16.0(15.4-17.4)$ & $0.2(-0.8$ to 1.2$)$ & 1408 \\
\hline \multicolumn{10}{|l|}{ Montana } \\
\hline \multirow[t]{2}{*}{ Decker } & GPPDS4 & 1984 & McRae loam/ & & & & & & \\
\hline & & & Midway silty clay loam & 45.11 & -106.93 & $300(277-316)$ & $15.7(14.1-17.3)$ & $-1.1(-2.8$ to 0$)$ & 1109 \\
\hline Miles City & GPPDS3 & 1994 & Havre loam & 46.39 & -105.89 & $296(247-324)$ & $15.4(13.5-16.1)$ & $0.5(-0.8$ to 1.4$)$ & 736 \\
\hline Miles City & GPPDS3 & 1999 & Havre loam & 46.39 & -105.89 & $306(271-363)$ & $15.8(15.1-16.7)$ & $1.3(0.5$ to 2.3$)$ & 736 \\
\hline \multicolumn{10}{|l|}{ Nebraska } \\
\hline Mead & PP5 & 1999 & Sharpsburg silt loam & 41.22 & -96.48 & $689(625-798)$ & $17.0(16.6-17.2)$ & 3.9 (3.6 to 4.3 ) & 364 \\
\hline Sidney & GPPDS4 & 1999 & Duroc loam & 41.38 & -103.00 & $428(274-528)$ & $17.1(16.8-17.7)$ & 1.7 (1.1 to 2.2$)$ & 1310 \\
\hline \multicolumn{10}{|l|}{ North Dakota } \\
\hline Mandan & GPPDS4 & 1999 & Parshall fine sandy loam & 46.80 & -100.77 & $474(286-643)$ & $12.9(12.5-13.3)$ & $-0.4(-0.8$ to 0.3$)$ & 510 \\
\hline \multicolumn{10}{|l|}{ Utah } \\
\hline Beaver & NUM6 & 2006 & Murdock silt loam & 38.35 & -112.59 & $312(273-377)$ & $17.0(16.6-17.5)$ & $0.8(0.4$ to 1.1$)$ & 1971 \\
\hline Blue Creek & IS5 & 1984 & Timpanogos silt loam & 41.93 & -112.44 & 397 (323-470) & $14.4(14.0-14.9)$ & $-1.1(-1.1$ to -0.7$)$ & 1564 \\
\hline Blue Creek & IS5 & 1989 & Timpanogos silt loam & 41.93 & -112.44 & $306(226-402)$ & $16.5(14.6-17.6)$ & $0.1(-1.1$ to 1.1$)$ & 1564 \\
\hline Blue Creek & IS5 & 1999 & Timpanogos silt loam & 41.93 & -112.44 & $313(278-356)$ & $16.8(15.8-17.4)$ & $0.7(-0.1$ to 1.7$)$ & 1564 \\
\hline Dugway & ISD6 & 1990 & Medburn fine sandy loam & 40.27 & -112.82 & 209 (169-245) & $17.4(16.2-18.4)$ & 2.1 (1.3 to 2.7$)$ & 1615 \\
\hline Monticello & ISD6 & 1984 & Begay fine sandy loam & 38.30 & -109.39 & $328(155-416)$ & $17.0(15.8-17.9)$ & 1.7 (1.3 to 2.5$)$ & 1879 \\
\hline \multirow[t]{2}{*}{ North Logan } & SRM4 & 1986 & Green Canyon gravelly loam/ & & & & & & \\
\hline & & & Parley's silt loam & 41.77 & -111.78 & $426(301-653)$ & $15.1(14.0-15.7)$ & 2.2 (1.7 to 2.7$)$ & 1509 \\
\hline \multirow[t]{2}{*}{ North Logan } & SRM4 & 1999 & Green Canyon gravelly loam/ & & & & & & \\
\hline & & & Parley's silt loam & 41.77 & -111.78 & $399(357-437)$ & $15.5(14.5-16.1)$ & $1.6(0.7$ to 3.0$)$ & 1509 \\
\hline Promontory & ISD5 & 2003 & Kearns silt loam & 41.67 & -112.48 & $310(216-404)$ & $17.1(16.2-18.0)$ & 2.2 (1.1 to 2.9$)$ & 1287 \\
\hline Scipio & NUM6 & 2003 & Unmapped & 39.20 & -112.24 & 397 (345-463) & $17.4(16.8-18.6)$ & 2.4 (1.4 to 3.2$)$ & 1772 \\
\hline Scipio & NUM6 & 2004 & Unmapped & 39.22 & -112.20 & $409(348-477)$ & $16.9(16.7-17.3)$ & $2.3(1.0$ to 3.3$)$ & 1831 \\
\hline Skull Valley \#1 & ISD6 & 1983 & Tooele fine sandy loam & 40.33 & -112.78 & $254(207-310)$ & $16.7(15.6-17.9)$ & 2.1 (1.1 to 2.9$)$ & 1435 \\
\hline Skull Valley \#2 & ISD6 & 1983 & Berent-Hiko Peak complex & 40.32 & -112.90 & $372(292-486)$ & $16.1(15.2-17.3)$ & $2.4(1.4$ to 3.5$)$ & 1689 \\
\hline Spring City & ISD5 & 2003 & Denmark gravelly loam & 39.51 & -111.53 & 274 (249-298) & $17.3(16.7-17.9)$ & $0.6(0.2$ to 1.0$)$ & 1734 \\
\hline \multicolumn{10}{|l|}{ Washington } \\
\hline Yakima & IS6 & 2002 & Selah silt loam & 46.85 & -120.37 & $264(177-347)$ & $15.2(14.9-15.6)$ & $2.2(1.5$ to 2.8$)$ & 699 \\
\hline \multicolumn{10}{|l|}{ Wyoming } \\
\hline \multirow[t]{2}{*}{ Guernsey } & GPPDS4 & 2002 & Deight-Thirtynine-Glendo & & & & & & \\
\hline & & & very fine sandy loam & 42.40 & -104.81 & $324(265-384)$ & $16.3(16.2-16.5)$ & $-0.1(-0.5$ to 0.3$)$ & 1494 \\
\hline \multirow[t]{2}{*}{ Guernsey } & GPPDS4 & 2004 & Mainter-Keeline & & & & & & \\
\hline & & & fine sandy loam & 42.25 & -104.73 & $316(248-401)$ & $17.7(16.8-18.1)$ & $0.5(-0.2$ to 0.8$)$ & 1323 \\
\hline Guernsey & GPPDS4 & 2005 & Mitchell very fine sandy loam & 42.24 & -104.74 & $312(248-401)$ & $17.8(16.8-18.1)$ & $0.5(-0.2$ to 0.8$)$ & 1396 \\
\hline Granger & IS4 & 1998 & Unmapped & 41.41 & -110.38 & $194(122-292)$ & $13.6(12.8-14.4)$ & $-2.8(-3.3$ to -2.0$)$ & 2101 \\
\hline \multirow[t]{2}{*}{ Soda Lake } & IS3 & 1991 & $\begin{array}{l}\text { Pinedale very bouldery- } \\
\text { Noclios, extremely }\end{array}$ & & & & & & \\
\hline & & & bouldery complex & 42.94 & -109.85 & $390(280-490)$ & $9.6(7.9-10.9)$ & $-6.3(-7.6$ to -5.7$)$ & 2360 \\
\hline
\end{tabular}

${ }^{1}$ PAR indicates Plant Adaptation Region (Vogel et al. 2005). 
Table 2. Mean stand establishment and 3-yr stand persistence values with corresponding standard errors in parentheses for cool-season perennial grass species evaluated in a varying number of studies across the Great Plains and Intermountain regions of the United States. Mean values followed by different letters significantly differ at $P \leq 0.05$.

\begin{tabular}{lcc}
\hline \multicolumn{1}{c}{ Species } & Stand persistence (\%) & Stand establishment (\%) \\
\hline Intermediate wheatgrass & $70(9) \mathrm{a}$ & $79(7) \mathrm{a}$ \\
Siberian wheatgrass & $69(8) \mathrm{a}$ & $70(6) \mathrm{a}-\mathrm{d}$ \\
Crested wheatgrass & $69(7) \mathrm{a}$ & $71(6) \mathrm{abc}$ \\
Tall wheatgrass & $66(12) \mathrm{a}$ & $75(9) \mathrm{ab}$ \\
Meadow brome & $64(12) \mathrm{a}$ & $69(9) \mathrm{a}-\mathrm{d}$ \\
Russian wildrye & $60(8) \mathrm{a}$ & $59(6) \mathrm{c}-\mathrm{g}$ \\
Western wheatgrass & $59(8) \mathrm{a}$ & $58(7) \mathrm{c}-\mathrm{g}$ \\
Smooth brome & $55(13) \mathrm{a}$ & $52(9) \mathrm{d}-\mathrm{h}$ \\
Bluebunch wheatgrass & $53(10) \mathrm{ab}$ & $57(7) \mathrm{d}-\mathrm{g}$ \\
RS wheatgrass & $52(10) \mathrm{ab}$ & $60(8) \mathrm{c}-\mathrm{f}$ \\
Thickspike wheatgrass & $51(9) \mathrm{abc}$ & $64(7) \mathrm{b}-\mathrm{e}$ \\
Snake River wheatgrass & $49(10) \mathrm{abc}$ & $52(9) \mathrm{d}-\mathrm{h}$ \\
Basin wildrye & $32(11) \mathrm{bcd}$ & $46(8) \mathrm{f}-\mathrm{h}$ \\
Slender wheatgrass & $29(12) \mathrm{cd}$ & $64(9) \mathrm{b}-\mathrm{e}$ \\
Altai wildrye & $29(11) \mathrm{cd}$ & $49(8) \mathrm{e}-\mathrm{h}$ \\
Squirreltail & $25(25) \mathrm{d}$ & $59(12) \mathrm{c}-\mathrm{g}$ \\
Indian ricegrass & $0(25) \mathrm{e}$ & $43(10) \mathrm{h}$ \\
\hline
\end{tabular}

ricegrass) and stand persistence values ranged from $70 \%$ (intermediate wheatgrass) to $0 \%$ (Indian ricegrass) (Table 2 ). Intermediate wheatgrass, tall wheatgrass $(75 \%)$, crested wheatgrass $(71 \%)$, Siberian wheatgrass $(70 \%)$, and meadow brome $(69 \%)$ possessed the highest stand establishment (Table 2 ). There were no significant differences among the 12 species with the largest stand persistence values (Table 2). Basin wildrye $(32 \%)$, Altai wildrye $(29 \%)$, slender wheatgrass $(29 \%)$, squirreltail $(25 \%)$, and Indian ricegrass $(0 \%)$ possessed lower stand persistence than the majority of the other species, and Indian ricegrass possessed the lowest stand persistence of any of the species (Table 2). Likely due to its rhizomatous nature, western wheatgrass was the only species that exhibited a marked increase of stand persistence (69\%) compared to stand establishment (58\%).

'Beefmaker' intermediate wheatgrass $(86 \%)$ possessed higher stand establishment than 48 of the individual varieties (Table 3 ). This included higher stand establishment than each of the varieties corresponding to Altai wildrye, bluebunch wheatgrass, basin wildrye, Indian ricegrass, RS wheatgrass, Russian wildrye, smooth brome, Snake River wheatgrass, squirreltail, thickspike wheatgrass, and western wheatgrass. 'NU-ARS AC2' crested wheatgrass $(80 \%)$ possessed higher stand persistence than 26 varieties, including all varieties corresponding to Altai wildrye, basin wildrye, Indian ricegrass, RS wheatgrass, slender wheatgrass, squirreltail, and thickspike wheatgrass (Table 3). 'Whitmar' bluebunch wheatgrass possessed stand persistence of $79 \%$, but was only measured for stand persistence at one location, which limited the inference of this result.

Intermediate wheatgrass, tall wheatgrass, crested wheatgrass, Siberian wheatgrass, and meadow brome possessed the best potential to maximize both stand establishment and stand persistence. Although the majority of the varieties corresponding to these species possessed high stand establishment and persistence, there were exceptions. For example, 'Douglas' crested wheatgrass did not possess high stand establishment or persistence. Thus, care must be taken to choose not only the appropriate species, but also the appropriate variety for revegetation.

\section{Influence of Climatic and Environmental Conditions}

Although results from the overall analysis across all sites provide a baseline for comparisons, the underlying interaction between sites and species and varieties resulted in confounded findings. The sites for this analysis comprise a wide range of climatic and environmental conditions and represented 12 plant adaptation regions (Table 1 ).

Stand establishment was correlated $(P \leq 0.05)$ with mean annual precipitation $(\mathrm{r}=0.42)$ and longitude $(0.46)$, and stand persistence was correlated with mean annual precipitation $(r=0.44)$, elevation $(r=-0.51)$, longitude $(0.49)$, and the year planted (0.63). Thus, more eastern, higher precipitation sites at lower elevations tended to have higher stand establishment and persistence values. For instance, the Mead, Nebraska site was the most eastern, received the highest precipitation, had the lowest elevation, and resulted in the highest stand establishment $(98 \%)$ and stand persistence $(83 \%)$ of any site. Although intuitively the most critical year would seem to be the establishment year, precipitation during the establishment at each site in this analysis was not associated with either stand establishment or stand persistence (data not shown). Because the correlation values were moderate to low, from a biological standpoint they suggested that environmental conditions affected stand establishment and persistence, but that they were not sufficient to explain differences in stand establishment and persistence.

\section{Site Precipitation}

The individual site results for each species are reported in Tables 4 and 5. However, due to the significant correlations between stand establishment and persistence and annual site precipitation, sites were partitioned into groups based on annual precipitation to allow further species performance inferences. The groups were low $(<310 \mathrm{~mm})$, intermediate (310-389 mm), and high (>389 $\mathrm{mm}$ ) annual precipitation.

Crested wheatgrass, Siberian wheatgrass, RS wheatgrass, Russian wildrye, and thickspike wheatgrass were among the species with the highest stand establishment at a majority of the low precipitation sites where they were evaluated (Table 4). Intermediate wheatgrass, tall wheatgrass, and thickspike wheatgrass were the only species evaluated at multiple low precipitation sites that were not among the species with the lowest stand establishment at any site. Indian ricegrass, Snake River wheatgrass, western wheatgrass, and Altai wildrye were among the species with the lowest stand establishment at all, or the majority, of the low precipitation sites where they were evaluated. Bluebunch wheatgrass, crested wheatgrass, intermediate wheatgrass, and Siberian wheatgrass possessed high stand establishment at the majority of intermediate precipitation sites where they were evaluated. Slender wheatgrass, Snake River wheatgrass, western wheatgrass, basin wildrye and Russian 
Table 3. Mean stand establishment and 3-yr stand persistence values with corresponding standard errors in parentheses for 69 cool-season perennial grass cultivars or prevariety germplasms evaluated in a varying number of evaluations across the Great Plains and Intermountain regions of the United States. Mean values followed by different letters significantly differ at $P \leq 0.05$

\begin{tabular}{|c|c|c|}
\hline Cultivar/Germplasm & $\begin{array}{c}\text { Stand } \\
\text { persistence (\%) }\end{array}$ & $\begin{array}{c}\text { Stand } \\
\text { establishment (\%) }\end{array}$ \\
\hline NU-ARSAC2 Crested wheatgrass & $80(10) \mathrm{a}$ & $74(9) a-i$ \\
\hline Whitmar Bluebunch wheatgrass & $79(28) \mathrm{a}$ & 45 (17) $\mathrm{p}-\mathrm{v}$ \\
\hline Recovery Western wheatgrass & $78(10) \mathrm{a}$ & 65 (9) c-m \\
\hline Beefmaker Intermediate wheatgrass & 77 (11) a & $86(9) \mathrm{a}$ \\
\hline Manska Intermediate wheatgrass & $76(11) a b$ & 79 (9) a-e \\
\hline Jose Tall wheatgrass & $76(13) a b$ & 79 (10) a-e \\
\hline Hycrest II Crested wheatgrass & $75(8) a b$ & $79(7) \mathrm{a}-\mathrm{e}$ \\
\hline CD II Crested wheatgrass & $75(9) a b$ & $80(7) a b c$ \\
\hline Hycrest Crested wheatgrass & $74(8) a b c$ & 77 (7) a-g \\
\hline Vavilov II Siberian wheatgrass & $74(10) a b c$ & $78(8) a-f$ \\
\hline Rush Intermediate wheatgrass & $74(11) a b c$ & $81(9) a b c$ \\
\hline Haymaker Intermediate wheatgrass & 73 (11) abc & $82(9) a b$ \\
\hline Flintlock Western wheatgrass & $72(10) a-d$ & $68(8) b-m$ \\
\hline Mankota Russian wildrye & $72(10) a-d$ & $66(8) b-m$ \\
\hline Oahe Intermediate wheatgrass & $71(11) \mathrm{a}-\mathrm{e}$ & $82(9) a b$ \\
\hline Cache Meadow brome & 71 (12) a-e & 75 (11) a-i \\
\hline Ephraim Crested wheatgrass & 70 (9) a-e & $64(8) d-n$ \\
\hline Manifest Intermediate wheatgrass & 70 (11) a-e & $78(9) a-f$ \\
\hline Fairway Crested wheatgrass & $69(9) a-f$ & $63(7)$ e-n \\
\hline Reliant Intermediate wheatgrass & 68 (11) a-g & $80(9) a b c$ \\
\hline P27 Siberian wheatgrass & 68 (9) $a-g$ & $59(7) \mathrm{i}-\mathrm{r}$ \\
\hline Arriba Western wheatgrass & 68 (9) $a-g$ & $55(8) 1-t$ \\
\hline Vavilov Siberian wheatgrass & $67(9) a-h$ & 70 (7) a-l \\
\hline Lincoln Smooth brome & 67 (14) a-h & $57(10) \mathrm{j}-\mathrm{s}$ \\
\hline Kazak Siberian wheatgrass & $66(9) \mathrm{a}-\mathrm{i}$ & $71(7) \mathrm{a}-\mathrm{I}$ \\
\hline Bozoisky II Russian wildrye & $65(8) a-i$ & $65(7) \mathrm{c}-\mathrm{m}$ \\
\hline Nordan Crested wheatgrass & $65(8) a-i$ & $64(7) d-n$ \\
\hline Rosana Western wheatgrass & 65 (8) a-i & $60(7) h-q$ \\
\hline Bozoisky-Select Russian wildrye & $65(8) a-i$ & $57(7) \mathrm{j}-\mathrm{s}$ \\
\hline Rodan Western wheatgrass & $65(9) a-i$ & $55(8) 1-t$ \\
\hline Roadcrest Crested wheatgrass & $64(10) a-j$ & $64(8) d-n$ \\
\hline Greenar Intermediate wheatgrass & $63(10) a-k$ & $70(8) a-1$ \\
\hline Tetra-1 Russian wildrye & $62(10) a-k$ & $67(8) b-m$ \\
\hline Barton Western wheatgrass & $62(11) a-k$ & $44(9) q-v$ \\
\hline Regar Meadow brome & 62 (13) a-k & $65(11) \mathrm{c}-\mathrm{m}$ \\
\hline Vinall Russian wildrye & $60(9) \mathrm{a}-\mathrm{I}$ & $53(8) m-u$ \\
\hline Alkar Tall wheatgrass & 60 (12) a-I & $73(9) a-j$ \\
\hline Platte Tall wheatgrass & 60 (13) a-l & $72(10) a-k$ \\
\hline Cabree Russian wildrye & 59 (13) a-I & $60(13) h-q$ \\
\hline Discovery Snake River wheatgrass & 59 (15) a-I & $62(14) f-0$ \\
\hline Tetracan Russian wildrye & $58(9) \mathrm{a}-\mathrm{I}$ & 57 (8) j-s \\
\hline Fleet Meadow brome & 57 (13) a-m & $68(11) b-m$ \\
\hline Luna Intermediate wheatgrass & $56(10) a-m$ & 77 (8) a-g \\
\hline Critana Thickspike wheatgrass & $52(10) b-n$ & $61(8) g-p$ \\
\hline NewHy RS wheatgrass & $52(10) b-n$ & $60(8) h-q$ \\
\hline Sodar Thickspike wheatgrass & $52(11) b-n$ & $64(8) d-n$ \\
\hline Douglas Crested wheatgrass & $50(9) c-n$ & $63(7)$ e-n \\
\hline Bannock Thickspike wheatgrass & $48(10) d-0$ & 67 (8) b-m \\
\hline Anatone Bluebunch wheatgrass & $47(10) \mathrm{e}-0$ & $64(8) d-n$ \\
\hline
\end{tabular}

Table 3. Continued.

\begin{tabular}{lll}
\hline \multicolumn{1}{c}{ Cultivar/Germplasm } & $\begin{array}{c}\text { Stand } \\
\text { persistence (\%) }\end{array}$ & $\begin{array}{c}\text { Stand } \\
\text { establishment (\%) }\end{array}$ \\
\hline Continental Basin wildrye & $45(14) \mathrm{f}-\mathrm{0}$ & $56(10) \mathrm{k}-\mathrm{s}$ \\
Mustang Altai wildrye & $44(13) \mathrm{g}-\mathrm{0}$ & $66(9) \mathrm{b}-\mathrm{m}$ \\
P7 Bluebunch wheatgrass & $43(10) \mathrm{h}-\mathrm{p}$ & $55(8) \mathrm{l}-\mathrm{t}$ \\
Firststrike Slender wheatgrass & $42(12) \mathrm{i}-\mathrm{p}$ & $76(10) \mathrm{a}-\mathrm{h}$ \\
Manchar Smooth brome & $42(13) \mathrm{i}-\mathrm{p}$ & $46(10) \mathrm{o}-\mathrm{v}$ \\
Fish Creek Bottlebrush squirreltail & $40(28) \mathrm{j}-\mathrm{p}$ & $62(15) \mathrm{f}-\mathrm{0}$ \\
Secar Snake River wheatgrass & $39(8) \mathrm{k}-\mathrm{q}$ & $42(7) \mathrm{s}-\mathrm{v}$ \\
Goldar Bluebunch wheatgrass & $37(9) \mathrm{l}-\mathrm{q}$ & $63(7) \mathrm{e}-\mathrm{n}$ \\
Swift Russian wildrye & $33(13) \mathrm{m}-\mathrm{r}$ & $41(10) \mathrm{s}-\mathrm{v}$ \\
Trailhead Basin wildrye & $31(11) \mathrm{n}-\mathrm{r}$ & $43(8) \mathrm{r}-\mathrm{v}$ \\
Pryor Slender wheatgrass & $24(12) \mathrm{o}-\mathrm{s}$ & $48(10) \mathrm{n}-\mathrm{v}$ \\
Prairieland Altai wildrye & $24(12) \mathrm{o}-\mathrm{s}$ & $32(9) \mathrm{vw}$ \\
Magnar Basin wildrye & $19(12) \mathrm{p}-\mathrm{s}$ & $38(9) \mathrm{uvw}$ \\
Pearl Altai wildrye & $19(13) \mathrm{p}-\mathrm{s}$ & $48(10) \mathrm{n}-\mathrm{v}$ \\
San Luis Slender wheatgrass & $19(15) \mathrm{p}-\mathrm{s}$ & $65(12) \mathrm{c}-\mathrm{m}$ \\
Toe Jam Creek Bottlebrush squirreltail & $19(28) \mathrm{p}-\mathrm{s}$ & $69(15) \mathrm{b}-\mathrm{m}$ \\
Sand Hollow Big squirreltail & $15(28) \mathrm{qrs}$ & $46(12) \mathrm{o}-\mathrm{v}$ \\
White River Indian ricegrass & $9(28) \mathrm{rs}$ & $66(15) \mathrm{b}-\mathrm{m}$ \\
Rimrock Indian ricegrass & $0(28) \mathrm{s}$ & $39(11) \mathrm{tuv}$ \\
Nezpar Indian ricegrass & $0(28) \mathrm{s}$ & $22(11) \mathrm{w}$ \\
\hline
\end{tabular}

wildrye possessed consistently low stand establishment at the intermediate precipitation sites. Many species possessed generally high stand establishment at the high precipitation sites. Nevertheless, smooth brome, Indian ricegrass, squirreltail, bluebunch wheatgrass, slender wheatgrass, Snake River wheatgrass, and the three wildrye species possessed low or moderate stand establishment at these sites.

For stand persistence, crested wheatgrass, Siberian wheatgrass, western wheatgrass, and Russian wildrye were the only species to possess consistently high values at the majority of low and intermediate precipitation sites (Table 5). Smooth brome, Indian ricegrass, bluebunch wheatgrass, slender wheatgrass, Snake River wheatgrass, Altai wildrye, and basin wildrye possessed consistently low stand persistence at the low precipitation sites. RS wheatgrass, Snake River wheatgrass, and basin wildrye were the only species to possess consistently low stand persistence at the intermediate precipitation sites. With the exceptions smooth brome, bluebunch wheatgrass, Snake River wheatgrass, Altai wildrye, and basin wildrye, the species all possessed generally high stand persistence at the high precipitation sites.

Although the site precipitation groupings indicated general trends in the data, not all sites followed these trends for a particular precipitation level (Tables 4 and 5). For instance, crested wheatgrass had low stand establishment at the Guernsey, Wyoming 2004 site. This was an intermediate precipitation site where, based on other similar sites, crested wheatgrass would have been expected to establish well. Additionally, the unbalanced nature of the data resulted in the evaluation of the different species at differing numbers of sites. Thus, some species were included at all sites in a particular precipitation grouping, whereas other species were 


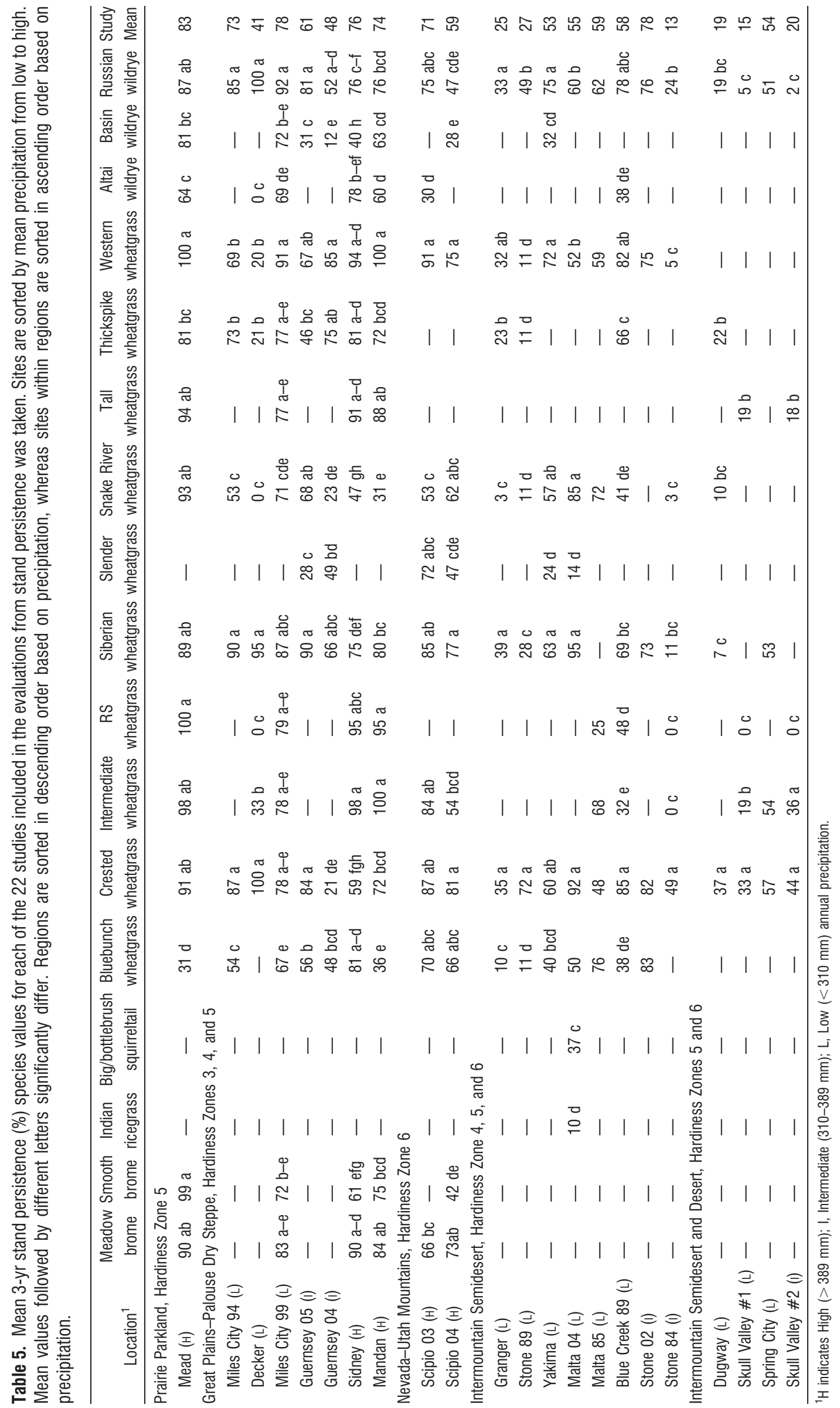


evaluated at only one or two of the sites in a particular grouping.

\section{Plant Adaptation Regions}

In conjunction with the grouping of sites by precipitation, the sites were also located in 12 PARs (Table 1). Results from individual evaluations are grouped under the corresponding PARs in Tables 4 and 5. Some trends concerning the appropriateness of the various species to PARs can be noted in the data. Crested, Siberian, thickspike, and intermediate wheatgrass are more widely adapted as indicated by their stand establishment values throughout the regions. Other species established well in some regions, but not in others. Meadow brome possessed consistently high stand establishment, but was only evaluated at two sites west of the Great Plains. Bluebunch wheatgrass, which is native to many of the Intermountain Semidesert sites, established well in this region, but outside the Guernsey, Wyoming sites possessed only low to moderate establishment in the other regions. RS, tall, thickspike, and western wheatgrass and Russian wildrye established well in the eastern regions and the North Logan sites, but established poorly elsewhere. Smooth brome, Indian ricegrass, squirreltail, Snake River wheatgrass, and Altai and basin wildrye possessed generally low establishment across the regions.

Crested and Siberian wheatgrass possessed high stand persistence across most of the regions. Meadow brome generally persisted well, but its evaluation in western regions was limited. Intermediate, RS wheatgrass, and tall wheatgrass persisted well in the eastern regions, but not in the western regions. Western wheatgrass and Russian wildrye persisted well in all regions but the Intermountain Semidesert and Desert. The stand persistence of western wheatgrass is consistent with its center of origin, corresponding to higher rainfall and humidity locations in the northern and central Great Plains, where many of the high precipitation sites were located. All remaining species rarely exhibited high persistence. Thus, some grasses are simply not adapted to some regions. As an example, and consistent with the precipitation groups, bluebunch wheatgrass, slender wheatgrass, Snake River wheatgrass, thickspike wheatgrass, and basin wildrye from the Intermountain West possessed low persistence in the eastern Great Plains as typified by the Mead, Nebraska location, probably due to root, crown, and foliar diseases that exist in high rainfall regions of the eastern Great Plains.

\section{Results of Selection}

Although differences were not always significant there was a strong trend for more recent plant releases to possess higher stand establishment than older releases of the same species. (Table 3). This is likely due to the limited evaluation and selection that accompanied the release of many of the older plant materials. However, the improvements in stand establishment did not necessarily correspond to improvements in stand persistence (Table 3). 'Mustang' Altai wildrye (66\%) possessed higher stand establishment than either of the of Altai wildryes, 'Pearl' $(48 \%)$ and 'Prairieland' (32\%). White River Indian ricegrass $(66 \%)$ possessed higher stand establishment than 'Rimrock' (39\%) or 'Nezpar' (22\%). 'Discovery' Snake River wheatgrass possessed higher stand establishment $(62 \%)$ than 'Secar' $(42 \%)$.

\section{DISCUSSION}

Successful revegetation of a site requires establishment of seeded species and subsequent growth by the species on available resources (reviewed in Seabloom et al. 2003). The synthesis of studies described herein addressed both of these requirements through the evaluation of species stand establishment and persistence under monoculture conditions. The 18 species and corresponding varieties evaluated herein represent the majority of perennial grass species currently or historically used in revegetation of degraded landscapes in the United States (e.g., US Department of the Interior-Bureau of Land Management 2009). Although few of the species possessed stand establishment values similar to species such as intermediate or crested wheatgrass, the majority established at values greater than $50 \%$ across all of the studies. However, species with stand establishment values less than $50 \%$ reflect revegetation sites with substantial areas of bare soil that remain unprotected from soil erosion and annual plant invasion. Nevertheless, only three species had stand establishment less than $50 \%$. Thus, most species established at acceptable levels, particularly at precipitation levels higher than $310 \mathrm{~mm}$ annual precipitation. Stand persistence was the trait for which more species fell short, particularly at low-precipitation sites. Therefore, the main distinction between the species evaluated in these studies was stand persistence rather than stand establishment. This result is consistent with earlier findings on a more limited number of species and sites (Bleak et al. 1965).

In a dynamic landscape where stands can reestablish from seedling recruitment, and populations successionally move from one perennial plant community to another, long-term persistence might not be that important. Moreover, if long-term persistence prevents successional progression of species on a site, it could be a negative. This is particularly true if greater plant diversity is desirable, such as in the case of assisted succession (Cox and Anderson 2004). Unfortunately, the data presented did not allow persistence of species in mixtures to be determined. However, in the case of many revegetation projects where potentially large seed banks of annual, invasive species exist (Humphrey and Schupp 2001), failure of revegetation species to persist for at least moderate amounts of time will lead to annual plant dominance and increased wildfire frequency. Therefore, on severely degraded sites with annual plant invasion and at low precipitation levels, the importance of longer-term persistence is of paramount importance. This can become an even more important consideration if plant adaptation profiles for specific ecoregions change due to the expected changes in climate. Site stability could be dependent upon having species at a site that have the broad adaptative capacity to be able to thrive under changing climatic conditions. Ultimately, land managers will need to determine the relative importance of stand persistence for their long-term site goals for biodiversity and their needs to balance soil stabilization and limit annual plant invasion. Public and private land managers make revegetation decisions based on criteria such as topography and size of the disturbance, seed prices, seed availability, and regulatory requirements (Richards et al. 1999). 
These results should provide further information to make more informed site decisions.

Mean annual precipitation and longitude were moderately correlated with stand establishment and persistence, although it is likely that precipitation and longitude are confounded due to the higher rainfall pattern of the more eastern sites. A larger proportion of the species had higher stand establishment and persistence at sites that received higher levels of precipitation. Thus, the results suggested, as has been noted previously (Bleak et al. 1965), that precipitation was the predominant environmental factor underlying stand establishment and persistence. Nevertheless, none of the correlations were sufficient to explain the differences in stand establishment and persistence among the species across the sites. It is likely that differences in stand establishment and persistence were due to inherent biological and genetic differences among the species and varieties, environmental conditions at the study sites, and interactions between biology/genetics and environment, or genotype by environment interaction (Falconer and Mackay 1996).

From a biological standpoint, differences in seed size and rhizomatous nature among the species were possible causes of response differences. The effect of seed size on seedling establishment is unclear. In some instances there has been evidence of correlation between large seed size and increased establishment (Lawrence 1963; Berdahl and Barker 1984), whereas in other instances there was no correlation (Waldron et al. 2006). Additionally, these studies encompassed only single species, rather than a comparison among species. Among the species with the largest seeds included in this study (Altai wildrye, meadow brome, RS wheatgrass, and tall wheatgrass) there was no obvious relationship to increased establishment. Altai wildrye and RS wheatgrass possessed generally low stand establishment values, and meadow brome and tall wheatgrass possessed generally high stand establishment values. On the other extreme, Siberian wheatgrass and Indian ricegrass were the species with the smallest seeds. Siberian wheatgrass possessed generally high stand establishment, but Indian ricegrass possessed low stand establishment.

When considering growth habit (Asay and Jensen 1996), the rhizomes of intermediate wheatgrass and western wheatgrass might have been beneficial for their stand persistence, but there appeared to be less benefit from rhizomes for the stand persistence of either RS or thickspike wheatgrass. Although it was unclear which growth or morphological traits correlated with stand establishment or persistence, there was evidence that the selection underlying the development of the newer varieties had in several instances resulted in increased stand establishment, which is consistent with previous selection studies for seedling establishment (Asay and Johnson 1980; Berdahl and Barker 1984; Waldron et al. 2006).

\section{IMPLICATIONS}

The evaluation of perennial, cool-season grass species for stand establishment and persistence provides an important baseline for land managers when making decisions for revegetation projects. The important findings for land managers are: 1) that many of the available perennial, cool-season grass species will result in acceptable establishment, particularly at higher precipitation sites; 2) at low precipitation sites there are fewer species that consistently produced adequate stands; and 3) the relative importance of stand persistence must be determined by the land managers when they determine priorities for biodiversity, soil stabilization, and invasive annual species competition.

\section{ACKNOWLEDGMENTS}

The authors gratefully acknowledge Kay Asay, Howard Horton, and Bryant Gomm (all retired), ARS, Logan, Utah; John Berdahl (retired), Agricultural Research Service (ARS), Mandan, North Dakota; Marshall Haferkamp (retired), ARS, Miles City, Montana; Jeff Lynn, Fort Carson, Colorado; Pete Nissen, Yakima Firing Center, Washington; and the range control staff at Camp Guernsey, Wyoming for their efforts in these evaluations.

\section{LITERATURE CITED}

Aguirre, L., And D. A. Johnson. 1991. Influence of temperature and cheatgrass competition on seedling development of two bunchgrasses. Journal of Range Management 44:347-354.

AsAY, K. H. 1992. Breeding potentials in perennial Triticeae grasses. Hereditas 116:167-173.

Asay, K. H., N. J. Chatterton, K. B. Jensen, T. A. Jones, B. L. Waldron, and W. H. HORTON. 2003. Breeding improved grasses for semiarid rangelands. Arid Land Research and Management 17:469-478.

Asay, K. H., W. H. Horton, K. B. Jensen, and A. J. Palazzo. 2001. Merits of native and introduced Triticeae grasses on semiarid rangelands. Canadian Journal of Plant Science 81:45-52.

Asay, K. H., and K. B. Jensen. 1996. Wheatgrasses. In L. E. Moser, D. R. Buxton, and M. D. Casler [EDS.]. Cool-season forage grasses. Agronomy Monograph 34. Madison: WI, USA: ASA-CSSA-SSSA Publishers. p. 691-724.

Asay, K. H., AND D. A. Johnson. 1980. Screening for improved stand establishment in Russian wild ryegrass. Canadian Journal of Plant Science 60:1171-1177.

Berdahl, J. D., AND R. E. Barker. 1984. Selection for improved seedling vigor in Russian wildrye. Canadian Journal of Plant Science 64:131-138.

Bleak, A. T., N. C. Frischknecht, A. P. Plummer, and R. E. Eckert, JR. 1965. Problems in artificial and natural revegetation of the arid shadscale vegetation zone of Utah and Nevada. Journal of Range Management 18:59-65.

Cox, R. D., and V. J. Anderson. 2004. Increasing native diversity of cheatgrassdominated rangeland through assisted succession. Journal of Range Management 57:203-210.

DoURLEIJN, C. J. 1993. Combining estimators from variety trials that are connected by control varieties only. Communications in statistics-theory and methods 22:1829-1847.

Falconer, D. S., AND T. F. C. Mackay. 1996. Introduction to quantitative genetics. 4th ed. Essex, UK: Addison Wesley Longman Limited. 464 p.

HumphreY, L. D., AND E. W. Schupp. 2001. Seed banks of Bromus tectorum-dominated communities in the Great Basin. Western North American Naturalist 61:85-92.

JoNEs, T. A. 1988. A probability method for comparing varieties against checks. Crop Science 28:907-912.

Jones, T. A., D. C. Nielsen, And J. R. Carlson. 1991. Developing a grazing-tolerant native grass for bluebunch wheatgrass sites. Rangelands 13:147-150.

LAWRENCE, T. 1963. A comparison of methods of evaluating Russian wild ryegrass for seedling vigor. Canadian Journal of Plant Science 43:307-312.

Norton, J. B., T. A. Monaco, J. M. Norton, D. A. Johnson, and T. A. Jones. 2004. Soil morphology and organic matter dynamics under cheatgrass and sagebrushsteppe plant communities. Journal of Arid Environments 57:445-466.

Norton, J. B., T. A. Monaco, and U. NoRton. 2007. Mediterranean annual grasses in western North America: kids in a candy store. Plant and Soil 298:1-5. 
Palazzo, A. J., K. B. Jensen, B. L. Waldron, and T. J. Cary. 2005. Effects of tank tracking on range grasses. Journal of Terramechanics 42:177-191.

PICKFoRD, G. D. 1932. The influence of continued heavy grazing and promiscuous burning on spring-fall ranges in Utah. Ecology 13:159-171.

Richards, R. T., J. C. Chambers, and C. Ross. 1999. Use of native plants on federal lands: policy and practice. Journal of Range Management 51:625-632.

Robins, J. G., B. L. Waldron, K. P. Vogel, J. D. Berdahl, M. R. Haferkamp, K. B. Jensen, T. A. Jones, R. Mitchell, and B. K. Kindiger. 2007. Characterization of testing locations for developing cool-season grass species. Crop Science 47:10041012.

Seabloom, E. W., W. S. Harpole, 0. J. Reichman, and D. Tllman. 2003. Invasion, competitive dominance, and resource use by exotic and native California grassland species. Proceedings of the National Academy of Sciences, USA 100:13384-13389.
Thacker, E., M. H. Ralphs, and T. A. Monaco. 2009. Seeding cool-season grasses to suppress broom snakeweed (Gutierrezia sarothrae), downy brome (Bromus tectorum), and weedy forbs. Invasive Plant Science and Management 2:237-246.

uS Department of the Interior-Bureau of Land Management. 2009. August 2009 seed buy. Available at: http://www.blm.gov/or/procurement/files/August_2009_Seed_ Purchase_Awards.pdf. Accessed 18 June 2010.

Voget, K. P., And R. A. Masters. 2001. Frequency grid-a simple tool for measuring grassland establishment. Journal of Range Management 54:653-655.

Vogel, K. P., M. R. Schmer, and R. B. Mitchell. 2005. Plant adaptation regions: ecological and climatic classifications of plant materials. Rangeland Ecology \& Management 58:315-319.

Waldron, B. L., J. G. Robins, K. B. Jensen, A. J. Palazzo, T. J. Cary, and J. D. Berdahl. 2006. Population and environmental effects on seed production, germination, and seedling vigor in western wheatgrass (Pascopyrum smithii [Rydb.] A. Löve). Crop Science 46:2503-2508. 